\title{
MicroRNA-214 regulates osteosarcoma survival and growth by directly targeting phosphatase and tensin homolog
}

\author{
XUMING WANG, JIABING SUN, CHUNJIANG FU, DEWEI WANG and ZHENGGANG BI
}

Department of Orthopedics, The First Affiliated Hospital of Harbin Medical University, Harbin, Heilongjiang 150001, P.R. China

Received December 2, 2013; Accepted July 1, 2014

DOI: $10.3892 / \mathrm{mmr} .2014 .2616$

\begin{abstract}
An increasing number of microRNAs (miRNAs) have been identified as diagnostic and prognostic biomarkers, as well as additional therapeutic tools, in skeletal diseases. Recent studies have established the pathophysiological role of miR-214, using human osteoporotic bone specimens. However, miR-214 expression levels and the underlying regulatory mechanism in human osteosarcoma remain unclear. Quantitative polymerase chain reaction (qPCR) was used to examine the expression of miR-214 in human osteosarcoma tissues and cells. Transfection of the cells with either a miR-214 expressing-plasmid, mimic or inhibitor was performed, in order to investigate the role of miR-214 in osteosarcoma. In this study, miR-214 was shown to be significantly increased in the majority of 15 examined osteosarcoma tissues and in the Saos-2 human osteosarcoma cell line. Overexpression of miR-214 in Saos-2 cells induced cell proliferation, while inhibition of miR-214 promoted Saos-2 cell apoptosis in vitro. Furthermore, ectopic expression of miR-214 markedly promoted osteosarcoma development in a subcutaneous xenotransplantation model in BALB/c athymic nude mice. The role of miR-214 in osteocarcinogenesis was further investigated and phosphatase and tensin homolog (PTEN) was determined to be a direct target of miR-214 in Saos-2 cells. The proliferation-promoting effect of PTEN knockdown was similar to that of miR-214 overexpression. This study revealed that miR-214 exerted a crucial role in promoting osteosarcoma progression and this suggests that modulation of miR-214 levels may provide a novel therapeutic approach in cancer treatment.
\end{abstract}

\section{Introduction}

Osteosarcoma (OS) is the most common type of primary malignant bone tumor arising from primitive transformed

Correspondence to: Dr Zhenggang Bi, Department of Orthopedics, The First Affiliated Hospital of Harbin Medical University, 23 Youzheng Street, Harbin, Heilongjiang 150001, P.R. China

E-mail: zhenggangbihyd@163.com

Key words: microRNA-214, phosphatase and tensin homolog, osteosarcoma, proliferation, apoptosis cells of mesenchymal origin that exhibit osteoblastic differentiation and produce malignant osteoid cells, and is associated with high morbidity among children and adolescents (1). The mechanisms underlying the pathogenesis and progression of OS have been analyzed for a number of years (2). However, the complex molecular etiology of OS remains poorly understood.

Emerging evidence has shown that microRNAs (miRNAs) are important in regulating different cellular processes by targeting numerous genes in human cancer. miRNAs are a class of endogenous non-coding RNAs (usually 18-25 nucleotides in length) that bind to the 3' untranslated region (3'-UTR) of specific target mRNAs, either preventing translation or causing destabilization and degradation at the posttranscriptional level (3). In specific cellular processes, miRNAs exert crucial functions, such as differentiation, morphogenesis and tumorigenesis (4). Accumulating evidence has shown that aberrant expression of miRNA is correlated with different types of human malignant tumor in terms of growth, progression, metastasis, and poorer prognosis (1,5-7); including OS (8). miRNAs may function as either oncogenes or tumor suppressor genes during tumorigenesis (8).

Recently, deregulated miR-214 has been shown to be associated with a variety of cancer types (5). The upregulation of miR-214 has been observed in ovarian cancer (9), gastric cancer (10) and nasopharyngeal carcinoma (11); by contrast, miR-214 expression was found to be downregulated in hepatocellular carcinoma $(12,13)$, breast cancer (14) and cervical cancer (15-17). These data suggest that the role of miR-214 is tumor-specific; thus in specific types of tumor, miR-214 may function as either an oncogene or a tumor suppressor, possibly dependent on the different miRNA targets in different cancer types. Notably, in a recent array-based screen of miRNAs involved in the pathogenesis of OS, miR-214 was identified to be upregulated in OS samples compared with control bone samples $(1,6)$. In another study, miR-214 was reported to be involved in the inhibition of osteoblast differentiation and osteoblastic bone formation in skeletal disorders (18). However, the functional role and the underlying mechanisms of miR-214 involvement in OS, have not been completely elucidated. Therefore, further investigation of the function and mechanism of miR-214 in OS is of great importance.

The aim of the present study was to investigate the function of miR-214 in OS survival and growth, identify the direct target protein regulated by $\mathrm{miR}-214$, and provide a potential therapeutic target for the treatment of OS. 


\section{Materials and methods}

Materials and reagents. The following monoclonal mouse antibodies were used in the present study: anti-phosphatase and tensin homolg (PTEN; A2B1), sc-7074; anti-AKT1/2/3 (BDI222), sc-56878; anti-phospho (p)-AKT (11E6), sc-81433; and anti- $\beta$-actin (C4), sc-47778. All of the antibodies were purchased from Santa Cruz Biotechnology, Inc. (Santa Cruz, CA, USA). The cell counting kit-8 (CCK-8) was obtained from Beyotime Institute of Biotechnology (Nantong, China). The inhibitor, mimics and non-specific negative control oligonucleotides for miR-214 and specific small interfering (si) RNA against human PTEN (5'-UUU GUC UCU GGU CCU UAC UUC C-3' and 5'-GGA AGT AAG GAC CAG AGA CAA A-3') were obtained from Guangzhou RiboBio Co., Ltd. (Guangzhou, China). All other chemicals and reagents were purchased from Sigma-Aldrich China, Inc. (Shanghai, China), unless otherwise specified.

Cell culture and tumor specimens. Saos-2 human OS cells were purchased from the American Type Culture Collection (ATCC; Manassas, VA, USA) and cultured in Dulbecco's modified Eagle's medium (DMEM; Gibco-BRL, Carlsbad, CA, USA) supplemented with $10 \%$ fetal bovine serum (FBS), $100 \mathrm{U} / \mathrm{ml}$ penicillin and $100 \mu \mathrm{g} / \mathrm{ml}$ streptomycin in a humidified $5 \% \mathrm{CO}_{2}$ incubator at $37^{\circ} \mathrm{C}$. The hFOB1.19 human osteoblastic cell line was also purchased from ATCC and was cultured in Ham's F12/DMEM supplemented with $10 \%$ FBS, $100 \mathrm{U} / \mathrm{ml}$ penicillin and $100 \mu \mathrm{g} / \mathrm{ml}$ streptomycin. A total of 15 human primary OS and matched adjacent noncancerous tissues were collected from patients attending the Affiliated Hospital of Harbin Medical University (Harbin, China). Tissue samples were frozen at $-80^{\circ} \mathrm{C}$ until the samples were used for RNA extraction. The human OS tissues were analyzed according to a procedure approved by the Clinical Research Committees of Harbin Medical University. All procedures were approved by the Local Research Ethics Committee of Harbin Medical School (Harbin, China). The study also conformed to the principles outlined in the Declaration of Helsinki. Written informed consent was obtained from all patients.

$R N A$ isolation and quantitative polymerase chain reaction $(q P C R)$. Total RNA was extracted using TRIzol reagent (Invitrogen, Carlsbad, CA, USA). For the detection of mature miR-214, RNA was reverse-transcribed using a specific reverse-transcription primer (Applied Biosystems, Inc., Foster City, CA, USA). The expression levels of miR-214 were quantified by qPCR using TaqMan miRNA assays (Applied Biosystems, Inc.), according to the manufacturer's instructions, with the Rotor-Gene 6000 system (Corbett Life Science, Qiagen, Hilden, Germany). The PCR assays for human mature miR-214 and human U6 small nuclear (sn)RNA were performed as follows: $95^{\circ} \mathrm{C}$ for $30 \mathrm{sec}, 40$ cycles of $95^{\circ} \mathrm{C}$ for $30 \mathrm{sec}, 60^{\circ} \mathrm{C}$ for $20 \mathrm{sec}$, and $70^{\circ} \mathrm{C}$ for $1 \mathrm{sec}$. The expression of U6 snRNA was used as an internal standard. The following RT primers were used: miR-214-RT, 5'-TCGTATCCAGTGCAGGGTCCGAGGTGCACTGGATACGACACTGCCTG-3'; and U6-RT, 5'-GTCGTATCCAGTGCAGGGTCCGAGGTATTCGCACTGG ATACGACAAAATATGGAAC-3'. The following PCR primers were used: U6: U6-Fwd, 5'-TGCGGGTGCTCGCTTCG-
GCAGC-3'; and reverse, 5'-CCAGTGCAGGGTCCGAGGT-3'; and miR-214: miR-214-Fwd, 5'-TGCGGACAGCAGGCACAGAC-3';and reverse, 5'-CCAGTGCAGGGTCCGAGGT-3'.

Vector construction and miRNA/small interfering (si)RNA transfection. miR-214 precursor sequences were released from full-length constructs with BamHI and EcoRI subcloned into the complementary sites of pcDNA3.1 (Invitrogen). Construction of the pcDNA3.1-miR-214 vector was performed as described previously $(15,16)$. The Saos- 2 cells were seeded in six-well plates at $30-50 \%$ confluency $24 \mathrm{~h}$ before transfection with the miR-214 mimics, or the empty parental vector as a control, using Lipofectamine 2000 reagent (Invitrogen) and stable transfectants were selected by growth in hygromycin.

Cell proliferation assay. At $24 \mathrm{~h}$ after transfection, the Saos- 2 cells were digested with trypsin and replated in 96-well plates. After $6 \mathrm{~h}$, the cells were labeled with $\mathrm{Br}-\mathrm{dU}$ for $18 \mathrm{~h}$. Subsequently, the level of Br-dU incorporation was determined using a Br-dU cell proliferation ELISA kit (Roche Diagnostics $\mathrm{GmbH}$, Penzberg, Germany). At 24 and $48 \mathrm{~h}$ after transfection, the Saos- 2 cells were incubated at $37^{\circ} \mathrm{C}$ in $10 \%$ CCK- 8 diluted in normal culture medium until visual color conversion occurred in the culture plates. The number of viable cells was assessed by absorbance at $450 \mathrm{~nm}$ using a microplate reader (Bio-Rad Laboratories, Inc., Hercules, CA, USA).

Flow cytometric analysis. The apoptosis assay was performed on the Saos- 2 cells $48 \mathrm{~h}$ after transfection with either miR-214 inhibitor or control using an Annexin V-fluorescein isothiocyanate apoptosis detection kit (KeyGen Biotech, Nanjing, China) and the cells were analyzed by fluorescence-activated cell sorting (FACS Scanner; BDi Biosciences, Franklin Lakes, NJ, USA).

Western blot analysis. For preparation of the cell extracts, the control cells and miR-214-transfected cells were washed thrice with ice-cold phosphate-buffered saline and lysed in lysis buffer, containing $50 \mathrm{mM}$ Tris- $\mathrm{HCl}(\mathrm{pH} 8.0), 150 \mathrm{mM}$ $\mathrm{NaCl}, 1 \mathrm{mM}$ EDTA, $1 \%$ Triton X-100 and PMSF $100 \mu \mathrm{g} / \mathrm{ml}$, on ice for $30 \mathrm{~min}$. Following centrifugation at $12,000 \mathrm{x} \mathrm{g}$ for $20 \mathrm{~min}$ at $4^{\circ} \mathrm{C}$, the supernatants were analyzed by $10 \%$ SDS-PAGE and then electrophoretically transferred to a nitrocellulose membrane (Invitrogen). After $2 \mathrm{~h}$ blocking with 5\% bovine serum albumin at room temperature, the membranes were incubated with anti-PTEN, anti-AKT, anti-P-AKT and anti- $\beta$-actin monoclonal antibodies, followed by incubation with horseradish peroxidase-conjugated goat anti-mouse immunoglobulin $\mathrm{G}$ polyclonal secondary antibodies (Pierce, Rockford, IL, USA). Protein bands were visualized with an enhanced chemiluminescence reagent (Pierce).

Tumor formation in nude mice. Nude mice (Vital River Laboratories, Beijing, China), aged four to six weeks, were subcutaneously injected with $1 \times 10^{6}$ Saos- 2 cells stably expressing high levels of miR-214. The mice were manipulated and housed according to procedures approved by the Local Medical Experimental Animal Care Commission. After five weeks, the mice were euthanized by cervical dislocation and tumor growth was examined. The tumor weight was measured using an electronic balance. The tumor volume (V) was 
A

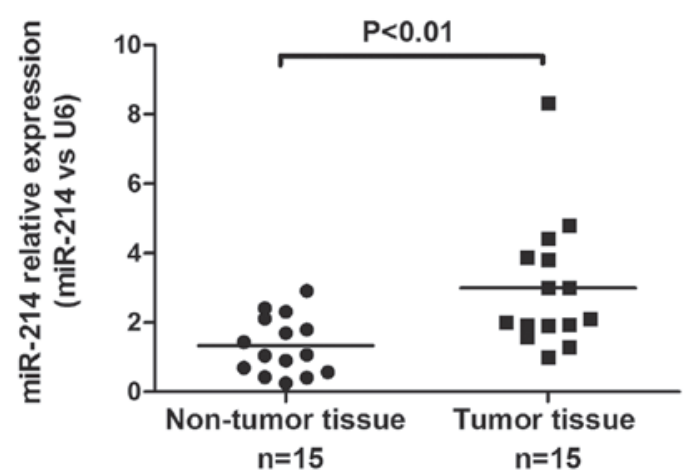

B

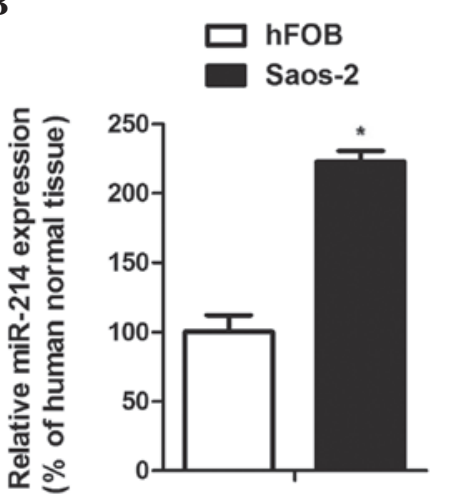

Figure 1. MicroRNA (miR)-214 is upregulated in osteosarcoma (OS) tissues and human OS cell lines. (A) Mature miR-214 expression levels were determined by reverse transcription-polymerase chain reaction analysis in the 15 pairs of OS and adjacent noncancerous tissues. U6 served as an internal control. (B) The relative expression levels of mature miR-214 in Saos-2 human OS cells and hFOB1.19 human osteoblastic cells were detected. ${ }^{\text {"P }}<0.05$ compared with hFOB.

A

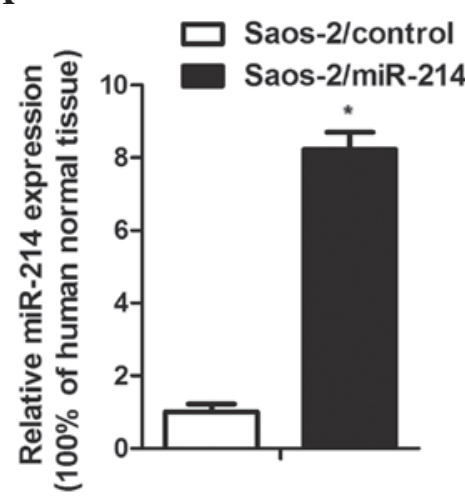

C
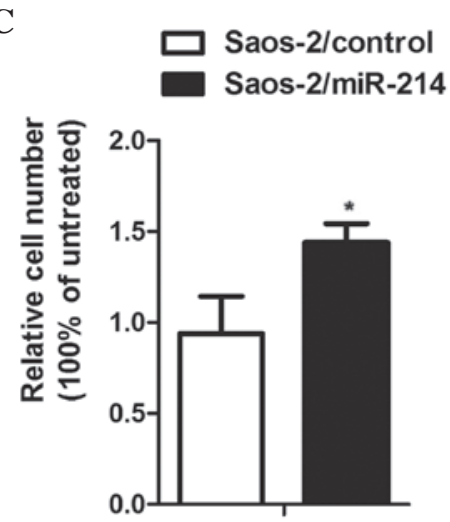

B

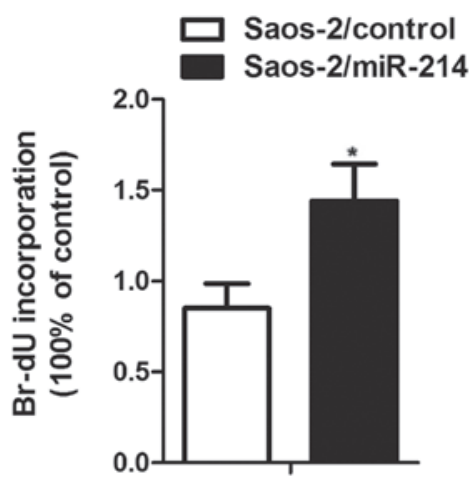

D

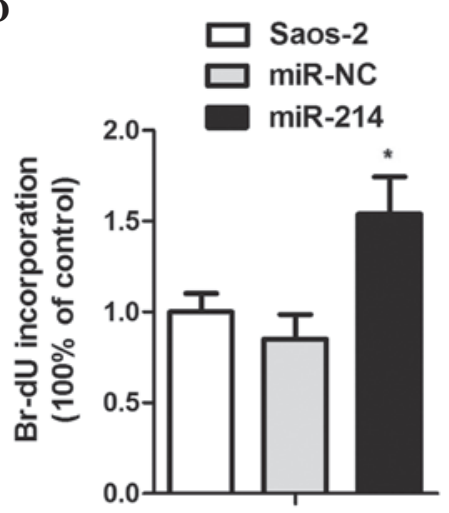

Figure 2. MicroRNA (miR)-214 overexpression promotes Saos-2 human osteosarcoma cell proliferation in vitro. (A) Successful overexpression of miR-214 was confirmed by reverse transcription-polymerase chain reaction following infection with miR-214-expressing plasmid, compared with transfection with vector control plasmid. (B) Overexpression of miR-214 accelerated cell proliferation in cells stably expressing miR-214, compared with control cells, as determined by Br-dU incorporation assay. (C) Overexpression of miR-214 accelerated cell proliferation in cells stably expressing miR-214, compared with control cells, as determined by cell counting kit-8 proliferation assay. (D) Transfection of miR-214 mimics accelerated cell proliferation in Saos-2 cells, compared with control cells, as determined by a Br-dU incorporation assay. ${ }^{\mathrm{P}} \mathrm{P}<0.05$ compared with control or miR-negative control (NC).

examined using a caliper and calculated using the following formula: $\mathrm{V}=\left(\mathrm{A} \times \mathrm{B}^{2}\right) / 2$; where $\mathrm{A}$ is the larger and $\mathrm{B}$ the smaller dimension of the tumor.

Statistical analysis. Two-tailed Student's t-test was used for statistical analysis. All data are presented as the mean \pm standard error of the mean. $\mathrm{P}<0.05$ was considered to indicate a statistically significant difference.

\section{Results}

miR-214 is upregulated in the human OS tissues and Saos-2 cells. To investigate the involvement of miR-214 in OS carcinogenesis, the expression levels of miR-214 in human OS tissues and Saos-2 human OS cells were analyzed by qPCR. The results revealed that the miR-214 expression levels were significantly increased in the human OS tissues compared with adjacent noncancerous 

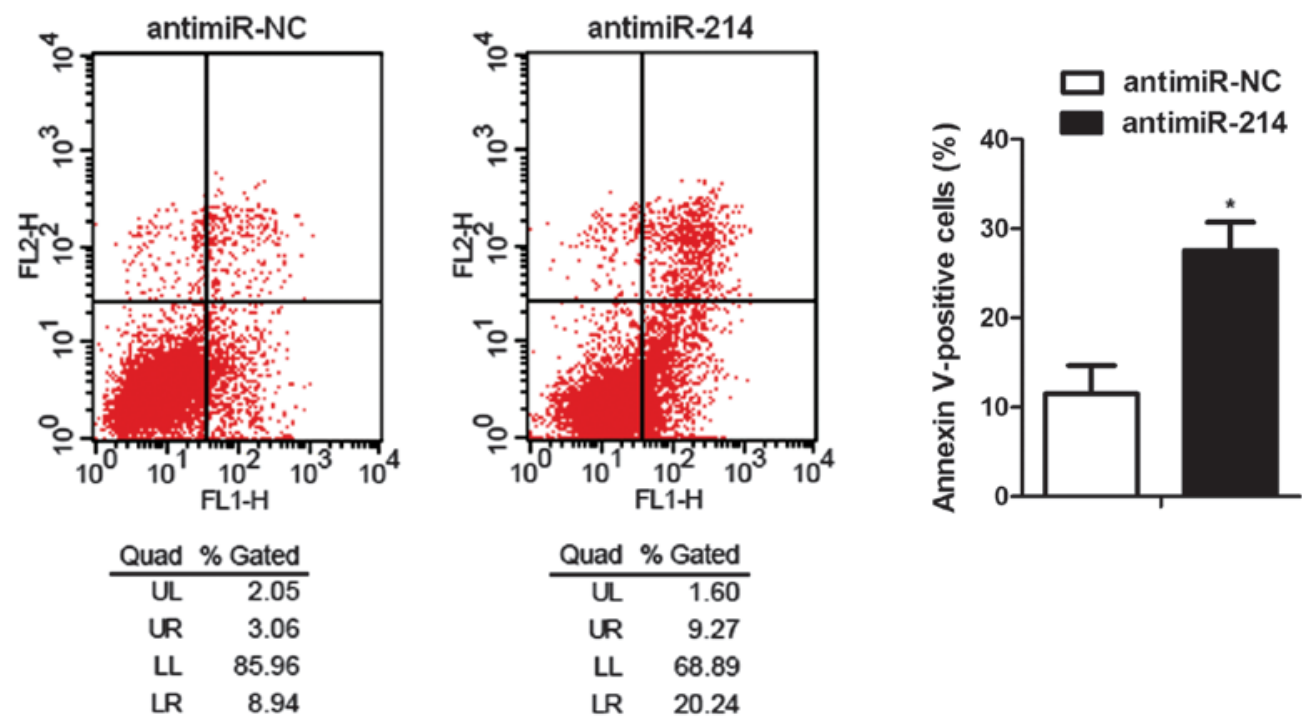

\begin{tabular}{cr} 
Quad & \% \\
\hline UL & 1.60 \\
UR & 9.27 \\
LL & 68.89 \\
UR & 20.24
\end{tabular}

Figure 3. MicroRNA (miR)-214 inhibits Saos-2 human osteosarcoma cell apoptosis in vitro. Saos-2 cells following treatment with miR-214 inhibitors exhibited increased cell apoptosis, compared with control cells, as measured by flow cytometric analysis. " $\mathrm{P}<0.05$ compared with anti-miR-negative control (NC).

A

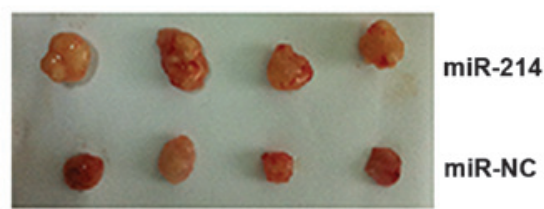

B

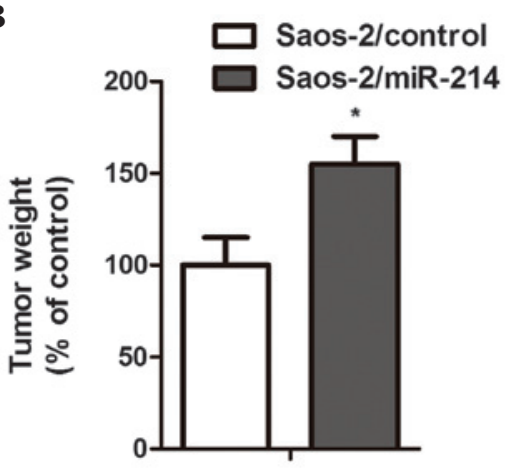

C

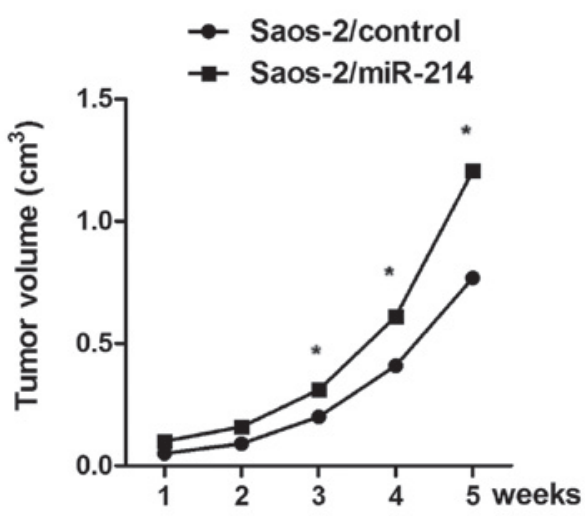

Figure 4. MicroRNA (miR)-214 overexpression promotes osteosarcoma (OS) tumorigenesis in vivo. (A) Representative tumors formed in nude mice injected with stable Saos-2 human OS cells transfected with either empty pcDNA3.1 plasmid (miR-NC) or pcDNA3.1-miR214 plasmid (miR-214). The Saos-2 human OS cells stably expressing miR-214 produced larger tumors than the control cells. (B) Tumor weights. The average tumor weight was significantly increased when miR-214 was overexpressed. (C) Growth curve of tumor volumes. Tumor volume was significantly increased by the overexpression of miR-214. ${ }^{*} \mathrm{P}<0.05$ compared with the control.

tissues $(\mathrm{P}<0.01$; Fig. 1A). Consistent with these observations, the Saos-2 human OS cells expressed significantly higher levels of miR-214 compared with the hFOB1.19 human osteoblastic cell line $(\mathrm{P}<0.05$; Fig. 1B). These data indicate that miR-214 may act as a novel regulator of OS development. Thus, it was decided to further investigate the role and function of miR-214 in OS.
miR-214 promotes Saos-2 cell proliferation in vitro. To examine the biological importance of miR-214 in OS tumorigenesis, Saos-2 cells stably expressing miR-214 were established using plasmids. Significantly increased miR-214 expression levels in these cells, compared with control cells, were confirmed by qPCR $(\mathrm{P}<0.05$; Fig. $2 \mathrm{~A})$. To further 
A

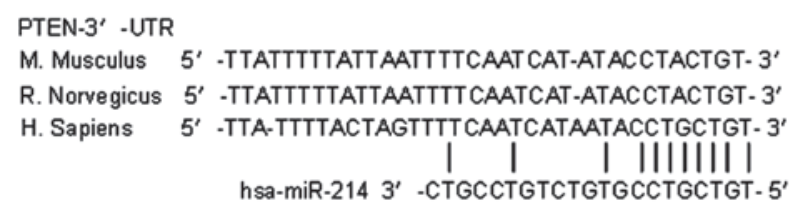

B

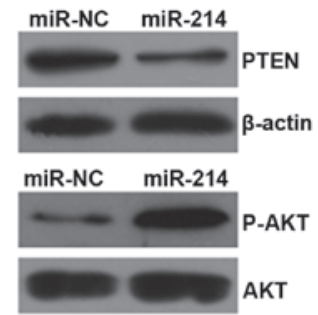

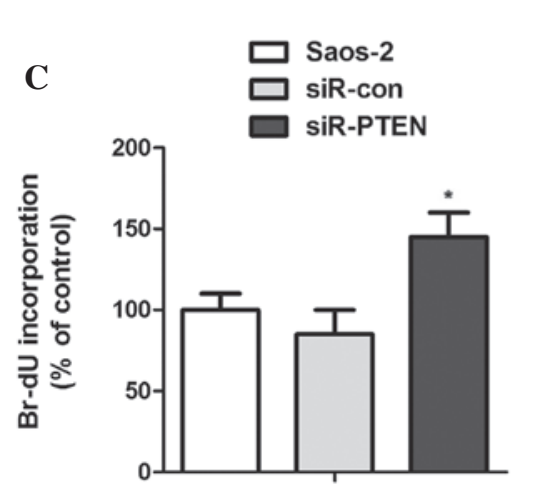

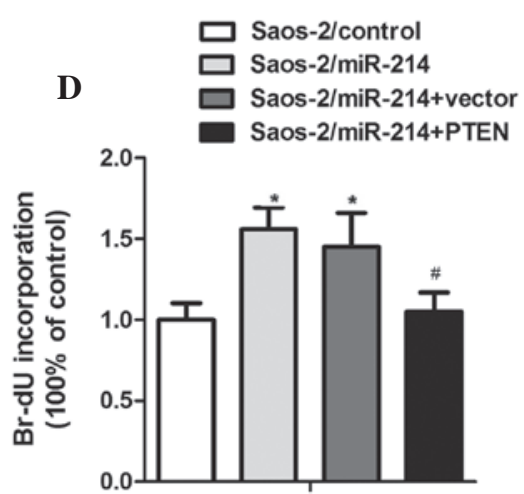

Figure 5. PTEN was a target of microRNA (miR)-214 in Saos-2 human osteosarcoma cells. (A) Bioinformatic analysis of PTEN 3' untranslated region (3'-UTR). Bioinformatic prediction using TargetScan revealed that the miR-214 binding site is conserved among different species. (B) Western blot analysis of PTEN, phospho (P)-AKT and AKT expression levels in Saos-2 control cells and those transfected with either empty pcDNA3.1 plasmid (miR-NC) or pcDNA3.1-miR214 plasmid (miR-214). The PTEN expression levels were normalized to those of $\beta$-actin. (C) Small interfering (si)RNA-mediated PTEN downregulation increased Saos-2 cell proliferation. (D) Restoration of PTEN reverses the oncogenic effects of miR-214 in Saos-2 cells. "P<0.05 compared with control, ${ }^{\#} \mathrm{P}<0.05$ compared with miR-214 + vector.

investigate whether overexpression of miR-214 increases the proliferation of Saos-2 cells, the cells were examined by $\mathrm{Br}-\mathrm{dU}$ incorporation and $\mathrm{CCK}-8$ proliferation assays. The two cell proliferation assays revealed that cell growth was significantly increased in Saos-2 cell lines stably expressing miR-214 compared with the corresponding control $(\mathrm{P}<0.05$; Fig. 2B and C). Similar results were observed in miR-214 mimic-transfected OS cells compared with either miR-control-transfected cells or untreated cells $(\mathrm{P}<0.05$; Fig. 2D). The results of these in vitro assays indicate that exogenous miR-214 significantly promoted the proliferation of OS cells.

miR-214 is an anti-apoptotic factor in Saos-2 cells in vitro. It was analyzed whether inhibition of miR-214 induces OS cell apoptosis and cell death using flow cytometric analysis to determine the number of early and late apoptotic Saos-2 cells following miR-214 inhibitor treatment (Fig. 3). As expected, few Annexin V-positive cells were detected in the miR-control-treated or untreated cells, whereas miR-214 inhibition significantly increased the percentage of apoptotic cells, compared with the control cells, as determined by Annexin V staining $(\mathrm{P}<0.05$; Fig. 3). These results indicate an antiapoptotic role of miR-214 in OS cells.

miR-214 enhances OS growth in vivo. Following the in vitro findings, animal experiments were conducted to further evaluate the effect of miR-214 on tumor growth in a subcutaneous xenotransplantation model with BALB/c athymic nude mice. Two groups of stably transfected cells (an Saos-2/control group, stable Saos-2 cells transfected with empty pcDNA3.1 plasmid; and a Saos-2/miR-214 group, stable Saos-2 cells transfected with pcDNA3.1-miR214 plasmid) were injected subcutaneously into the right flank of the nude mice as described. After five weeks, the mice were sacrificed and the size of the tumor nodules was examined. As expected, the mice injected with stable Saos-2 cells transfected with pcDNA3.1-miR214 exhibited a significant increase in tumor weight and tumor volume compared with the controls from three weeks onwards $(\mathrm{P}<0.05$; Fig. 4A-C). These results further suggest that miR-214 may function as a tumor oncogenic miRNA in OS cells.

miR-214 targets PTEN resulting in activation of the AKT signaling pathway. To analyze the underlying mechanism by which miR-214 promotes the growth of OS cells, bioinformatic analysis was performed to search for genes regulated by miR-214 using the TargetScan software program (http://www. targetscan.org). Following a preliminary screen, the miR-214 binding site located on the 3'-UTR of PTEN was found to be conserved in different species (Fig. 5A). Furthermore, a previous study reported that miR-214 suppresses PTEN expression in human ovarian tumor cells (9). Thus, PTEN was investigated as a possible target of miR-214; the effect of miR-214 on the endogenous expression levels of PTEN was examined in the Saos- 2 cells. As expected, the western blot analysis demonstrated that transfection with miR-214 mimics reduced the PTEN protein expression levels in Saos- 2 cells. Furthermore, miR-214 overexpression was found to increase the protein levels of P-AKT (Fig. 5B). These results suggest that PTEN is a target of miR-214 in OS cells. 
PTEN has been previously demonstrated to modulate the tumor suppressor response in human ovarian tumors in vivo (9). To examine the effect of PTEN on OS cell proliferation, a specific siRNA against human or human PTEN was transfected into the Saos-2 cells. The siRNA-mediated PTEN downregulation was found to significantly increase Saos-2 cell proliferation, compared with those of the two control cell groups $(\mathrm{P}<0.05$; Fig. 5C). These results suggest that the proliferation-promoting effect of PTEN knockdown was similar to that of miR-214 overexpression. Subsequently, it was determined whether restoration of PTEN reverses the oncogenic effects of miR-214. Transfection of pcDNA3.1-PTEN into Saos-2 cells stably expressing miR-214 efficiently restored PTEN expression levels and reversed the increased cell proliferation effect induced by miR-214 (Fig. 5D).

In conclusion, these results indicate that miR-214 inhibits PTEN translation, resulting in activation of the AKT signaling pathway, thus promoting OS tumor survival and growth.

\section{Discussion}

miR-214 is located in Dynamin 3 opposite strand (Dnm3os), a gene transcribed into a non-coding RNA, of which the expression is induced by the transcription factor Twist-1 in mice and humans (19,20). Increasing evidence indicates that miR-214 may be key in human cancer, and is involved in carcinogenesis, tumor progression and metastasis (5). For instance, miR-214 was shown to contribute to melanoma tumor progression through suppression of TFAP2C and promote tumorigenesis by targeting lactotransferrin in nasopharyngeal carcinoma $(5,11)$. However, the role of miR-214 in OS genesis remains unknown. Predicting and identifying the miR-214-targeting genes offers an experimental basis for further investigation into the regulatory mechanism of miR-214 in OS cells. In fact, an increasing number of miR-214 targets, including MEK3, JNK1, Plexin-B1, Ezh2, XBP-1, PDGF and TFAP2C, have been characterized in various tumor types, such as ovarian cancer, liver cancer, cervical cancer and melanoma $(12,13,16)$. In the present study, miR-214 was predicted and confirmed to act as a tumorigenesis factor in OS through targeting the 3'-UTR of PTEN.

PTEN is a tumor suppressor with sequence homology to protein tyrosine phosphatases and the cytoskeletal protein tensin, and specifically inhibits tumorigenesis by negatively regulating the phosphoinositide 3-kinase/AKT signaling pathway (9). The most recently identified PTEN tumor suppressor gene has been determined to be defective in a large number of human cancer types (21). Recent studies have emphasized the causative association between miRNA deregulation and PTEN deficiency in tumor development (9). Studies have demonstrated that PTEN is controlled by multiple miRNAs, including miR-21, miR-26a, miR-216a and miR-217 (22-25). In a recent study, TargetScan was used and indicated that PTEN was a potential target gene of miR-214 (26). In the present study, the effects of miR-214 on PTEN expression levels and AKT activation in Saos-2 human OS cells were investigated. miR-214 was found to inhibit PTEN expression, increase AKT phosphorylation, induce cell proliferation and inhibit apoptosis in Saos-2 cells in vitro, and promote tumor formation in vivo. Notably, previous studies have shown that ectopic expression of miR-214 in miR-214-negative ovarian cancer cells inhibited wild-type PTEN-3'-UTR but not mutated PTEN-3'-UTR reporter activity (9). The data from the present study are consistent with the results of other studies, and also demonstrated that miR-214 directly targeted PTEN in OS cells, promoted OS cell proliferation and propagated OS tumor development.

In addition, miR-214 is involved in the regulation of the quantity of ATF4 protein in bone tissues, and the inhibition of osteoblast differentiation and osteoblastic bone formation in skeletal disorders. Notably, osteoblast-specific manipulation of miR-214 levels by miR-214 antagomir treatment in miR-214 transgenic, ovariectomized or hind limb-unloaded mice revealed an inhibitory role of miR-214 in regulating bone formation, which provides a novel therapeutic approach for skeletal disorders (18). As miR-214 was revealed in the present study to be upregulated in the human OS cancer tissues and function as an oncogenic miRNA in OS cells, OS cell-specific modulation of miR-214 expression by miR-214 antagomir treatment may provide a novel therapeutic approach for human OS and may become possible in clinical practice. Further understanding of the molecular mechanism by which miR-214 functions may provide novel avenues of investigation to aid early diagnosis and treatment of this highly malignant tumor.

In conclusion, the present study demonstrated that miR-214 was upregulated in OS cells and tissues, and the ectopic expression of miR-214 induced cell proliferation and inhibited cell apoptosis. The tumorigenetic function of miR-214 was mediated by the downregulation of the downstream target gene PTEN. The data suggest that the pharmacological manipulation of miR-214 expression may be a novel tool in OS treatment.

\section{Acknowledgements}

This study was supported by a grant from the National Science Foundation of China (grant no. 81171692).

\section{References}

1. Jones KB, Salah Z, Del Mare S, et al: miRNA signatures associate with pathogenesis and progression of osteosarcoma. Cancer Res 72: 1865-1877, 2012.

2. Liu JJ, Liu S, Wang JG, et al: Telangiectatic osteosarcoma: a review of literature. Onco Targets Ther 6: 593-602, 2013.

3. Bartel DP: MicroRNAs: genomics, biogenesis, mechanism, and function. Cell 116: 281-297, 2004.

4. Valencia-Sanchez MA, Liu J, Hannon GJ and Parker R: Control of translation and mRNA degradation by miRNAs and siRNAs. Genes Dev 20: 515-524, 2006.

5. Penna E, Orso F, Cimino D, et al: microRNA-214 contributes to melanoma tumour progression through suppression of TFAP2C. EMBO J 30: 1990-2007, 2011.

6. Wang Z, Cai H,Lin L, Tang M and Cai H: Upregulated expression of microRNA-214 is linked to tumor progression and adverse prognosis in pediatric osteosarcoma. Pediatr Blood Cancer 61: 206-210, 2014.

7. Zhou X, Wei M and Wang W: MicroRNA-340 suppresses osteosarcoma tumor growth and metastasis by directly targeting ROCK1. Biochem Biophys Res Commun 437: 653-658, 2013

8. Miao J, Wu S, Peng Z, Tania M and Zhang C: MicroRNAs in osteosarcoma: diagnostic and therapeutic aspects. Tumour Biol 34: 2093-2098, 2013.

9. Yang H, Kong W, He L, et al: MicroRNA expression profiling in human ovarian cancer: miR-214 induces cell survival and cisplatin resistance by targeting PTEN. Cancer Res 68: 425-433, 2008.

10. Ueda T, Volinia S, Okumura H, et al: Relation between microRNA expression and progression and prognosis of gastric cancer: a microRNA expression analysis. Lancet Oncol 11: 136-146, 2010

11. Deng M, Ye Q, Qin Z, et al: miR-214 promotes tumorigenesis by targeting lactotransferrin in nasopharyngeal carcinoma. Tumour Biol 34: 1793-1800, 2013. 
12. Duan Q, Wang X, Gong W, et al: ER stress negatively modulates the expression of the miR-199a/214 cluster to regulates tumor survival and progression in human hepatocellular cancer. PLoS One 7: e31518, 2012.

13. Shih TC, Tien YJ, Wen CJ, et al: MicroRNA-214 downregulation contributes to tumor angiogenesis by inducing secretion of the hepatoma-derived growth factor in human hepatoma. J Hepatol 57: 584-591, 2012.

14. Derfoul A, Juan AH, Difilippantonio MJ, Palanisamy N, Ried T and Sartorelli V: Decreased microRNA-214 levels in breast cancer cells coincides with increased cell proliferation, invasion and accumulation of the Polycomb Ezh2 methyltransferase. Carcinogenesis 32: 1607-1614, 2011.

15. Qiang R, Wang F, Shi LY, et al: Plexin-B1 is a target of miR-214 in cervical cancer and promotes the growth and invasion of $\mathrm{HeLa}$ cells. Int J Biochem Cell Biol 43: 632-641, 2011.

16. Yang Z, Chen S, Luan X, et al: MicroRNA-214 is aberrantly expressed in cervical cancers and inhibits the growth of HeLa cells. IUBMB Life 61: 1075-1082, 2009

17. Peng RQ, Wan HY, Li HF, Liu M, Li X and Tang H: MicroRNA-214 suppresses growth and invasiveness of cervical cancer cells by targeting UDP-N-acetyl-alpha-D-galactosamine :polypeptide $\mathrm{N}$-acetylgalactosaminyltransferase 7. J Biol Chem 287: 14301-14309, 2012.

18. Wang X, Guo B, Li Q, et al: miR-214 targets ATF4 to inhibit bone formation. Nat Med 19:93-100, 2013.
19. Lee YB, Bantounas I, Lee DY, Phylactou L, Caldwell MA and Uney JB: Twist-1 regulates the miR-199a/214 cluster during development. Nucleic Acids Res 37: 123-128, 2009.

20. Sakurai K, Furukawa C, Haraguchi T, et al: MicroRNAs miR-199a-5p and $-3 p$ target the Brm subunit of SWI/SNF to generate a double-negative feedback loop in a variety of human cancers. Cancer Res 71: 1680-1689, 2011.

21. Zhao G, Cai C, Yang T, et al: MicroRNA-221 induces cell survival and cisplatin resistance through PI3K/Akt pathway in human osteosarcoma. PLoS One 8: e53906, 2013.

22. Guo P, Nie Q, Lan J, Ge J, Qiu Y and Mao Q: C-Myc negatively controls the tumor suppressor PTEN by upregulating miR-26a in glioblastoma multiforme cells. Biochem Biophys Res Commun 441: 186-190, 2013.

23. Tian L, Fang YX, Xue JL and Chen JZ: Four MicroRNAs promote prostate cell proliferation with regulation of PTEN and its downstream signals in vitro. PLoS One 8: e75885, 2013.

24. Wang D, Zhang H,Li M, et al: MicroRNA-124 controls the proliferative, migratory, and inflammatory phenotype of pulmonary vascular fibroblasts. Circ Res 114: 67-78, 2014.

25. Wang Y, Gao X, Wei F, et al: Diagnostic and prognostic value of circulating miR-21 for cancer: A systematic review and meta-analysis. Gene 533: 389-397, 2014.

26. Zhang ZC, Li YY, Wang HY, et al: Knockdown of miR-214 promotes apoptosis and inhibits cell proliferation in nasopharyngeal carcinoma. PloS One 9: e86149, 2014. 